\title{
Crowdsourcing in China: Exploring the Work Experiences of Solo Crowdworkers and Crowdfarm Workers
}

\author{
Yihong Wang \\ $\mathrm{Xi}$ 'an Jiaotong-Liverpool \\ University \\ China
}

Yihong.Wang18@student.xjtlu.edu.cn

\author{
Alan Chamberlain \\ Univerity of Nottingham \\ United Kingdom \\ Alan.Chamberlain@nottingham.ac.uk
}

\author{
Michael Saker \\ City, University London \\ United Kingdom \\ Michael.Saker@city.ac.uk
}

\author{
Ioanna Lykourentzou \\ Netherlands \\ I.Lykourentzou@uu.nl
}

\author{
Vassilis-Javed Khan \\ Eindhoven University of \\ Technology \\ Netherlands \\ V.J.Khan@tue.nl
}

\begin{abstract}
Recent research highlights the potential of crowdsourcing in China. Yet very few studies explore the workplace context and experiences of Chinese crowdworkers. Those that do, focus mainly on the work experiences of solo crowdworkers but do not deal with issues pertaining to the substantial amount of people working in 'crowdfarms'. This article addresses this gap as one of its primary concerns. Drawing on a study that involves 48 participants, our research explores, compares and contrasts the work experiences of solo crowdworkers to those of crowdfarm workers. Our findings illustrate that the work experiences and context of the solo workers and crowdfarm workers are substantially different, with regards to their motivations, the ways they engage with crowdsourcing, the tasks they work on, and the crowdsourcing platforms they utilize. Overall, our study contributes to furthering the understandings on the work experiences of crowdworkers in China.
\end{abstract}

\section{Author Keywords}

Crowdsourcing; Work Experience; Crowdworkers;

Crowdfarms; Reputation; Work Life Balance; Tasks; Platform Satisfaction; Motivations and Attitudes

\section{CCS Concepts \\ -Information systems $\rightarrow$ Crowdsourcing;}

\section{INTRODUCTION}

Crowdsourcing, i.e. the process of outsourcing tasks by organizations or individuals online in the form of an 'open-call', has become an international phenomenon attracting businesses and a crowd workforce across the globe [19, 41]. China, for

Permission to make digital or hard copies of all or part of this work for personal or classroom use is granted without fee provided that copies are not made or distributed for profit or commercial advantage and that copies bear this notice and the full citation on the first page. Copyrights for components of this work owned by others than the author(s) must be honored. Abstracting with credit is permitted. To copy otherwise, or republish, to post on servers or to redistribute to lists, requires prior specific permission and/or a fee. Request permissions from permissions@ acm.org.

CHI '20, April 25-30, 2020, Honolulu, HI, USA.

(C) 2020 Association for Computing Machinery.

ACM ISBN 978-1-4503-6708-0/20/04 \$15.00.

http://dx.doi.org/10.1145/3313831.3376473 instance, is one of the world's most populous countries and has a rapidly growing digital economy that now supplies a substantial workforce to crowdsourcing platforms [1]. Subsequently, crowdsourcing is regarded as a new value creation model, which has invigorated China's IT industries leveraging human intelligence $[60,63]$. According to Huo, Zheng and Tu [47], by 2017 there were already 30 million Chinese crowdworkers serving more than 190,000 enterprises and individuals worldwide, generating a total business turnover of CNY 5 billion (approx. US\$ 700M).

At the time of writing this article, $\mathrm{ZBJ}^{1}$ and $\mathrm{Epwk}^{2}$ have established themselves as two of the most prominent crowdsourcing platforms in China with around 19 million active crowdworkers respectively. ZBJ and Epwk cover an array of crowdsourcing tasks ranging from click-work to logo and product design. For the most part, these platforms operate in a similar manner to other - perhaps more known in the Western world - crowdsourcing platforms, such as Upwork ${ }^{3}$ and Amazon Mechanical Turk ${ }^{4}$.

Although studies exploring crowdsourcing and the work experiences of crowdworkers in various countries are becoming increasingly prevalent in CSCW and HCI literature (e.g. [42, 17]), only a handful of studies have specifically focused on Chinese crowdworkers. These studies have mainly focused on how crowdworkers select and complete tasks, demonstrating that the crowdworkers in the Chinese platforms are predominantly individuals seeking to earn additional income in their spare time ${ }^{5}$. For example, To and Lai [51] found that Chinese crowdworkers display a strong willingness to do creative tasks, and that they would crowdwork with relatively lower payouts than crowdworkers in Western countries. Developing

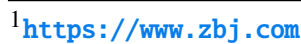

${ }^{2}$ https://epwk. com

${ }^{3}$ https: //WwW . upwork. com/

${ }^{4}$ https://www . mturk. com/

5 http: //Ww . chinadaily . com. cn/business/2017-10/30/ content_33898676.htm
} 
this point further, Yang et al. [62]suggest that Chinese crowdworkers tend to target tasks with fewer participants so as to increase their opportunities of winning bids, and that they have a propensity to select tasks with higher expected rewards.

While these studies are helpful, in the main, they do not provide sufficient understanding about the work experience of Chinese crowdworkers because they do not purposefully engage with key aspects of this work experience, such as motivations and attitudes, crowdwork satisfaction, work/life balance etc. Moreover, they do not take into account recent findings that illustrate the increasingly important role "crowdfarms" ie. companies that hire individuals on salary to perform crowdtasks they procure from various crowdsourcing platforms now play in Chinese platforms [55]. In contrast with Chinese solo crowdworkers who are found to usually work from home alone and undertake simple and easy tasks [56, 62, 48], crowdfarm workers work in offices and do large and complex tasks together in teams. In addition, as the salaried employees in companies, crowdfarm workers also work under the supervision of the managers who are in charge of procuring tasks, communicating with requesters and, oftentimes, performing the detailed tasks as well. As prior work shows that the characteristics of tasks [10], work environment [30] and team structure [65] affect crowdworkers' experience and behavior, one would expect more studies in the literature that investigate the different work experiences between solo crowdworkers and crowdfarm workers in crowdsourcing context in China.

To address this gap, our paper reports on 25 interviews with solo crowdworkers and 23 crowdfarm workers affiliated with the largest Chinese crowdsourcing platform, namely ZBJ. The interviews focus on the work experience of solo crowdworkers and crowdfarm workers and explore the following themes: (1) work environment, (2) tasks, (3) motivation and attitudes, (4) rewards, (5) reputation, (6) crowdwork satisfaction, and (7) work/life balance.

A significant contribution of this paper to both the CSCW and HCI communities, therefore, is that it represents one of the first scholarly investigations into the work experiences of a new type of crowdworker in the crowdsourcing context of China: crowdfarm workers. In addition, this paper also casts valuable light on the phenomenological differences between solo Chinese crowdworkers and crowdfarm workers by comparing and contrasting their lived experience through the thematic schema detailed above. Based on these novel findings, our paper suggests crowdsourcing platforms should not only be designed with the needs of solo crowdworkers but also the different characteristics of crowdfarm workers. For example, extending the platform communication systems and integrating them with popular Chinese social media (e.g. Wechat) is beneficial in large and complex tasks where constant feedback and coordinations are needed.

The rest of this paper is organised in the following way. First, we provide a review of relevant literature, while developing the thematic approach that underpins this research. Following this, we outline the methodological design of this study and explain how our data was analysed. We then present our findings, while contextualising the work experience of the solo and crowdfarm workers through a recourse to our thematic schema. We then conclude this project with a discussion of our findings, before reflecting on the limitations of this research, and providing suggestions for future work.

\section{BACKGROUND}

Since Jeff Howe initially coined the term "crowdsourcing" in 2006 to label the practice of leveraging a large, undefined and anonymous group of people to perform online tasks outsourced by individuals and organizations [19], the definition of crowdsourcing has continued to develop and taken on new contours. Today, definitions of crowdsourcing frequently involve some or all of the following element: (1) a problem-solving tool [7], (2) an online distributed problem-solving and production model [3, 8, 38], (3) an open collaborative learning paradigm [52], and (4) a new resource for product development [40]. By extracting the common elements from over 40 different kinds of crowdsourcing definitions, Estelles-Arolas and Gonzalez-Ladron-de-Guevarara [14] have helpfully summarized and defined crowdsourcing as a distributed problemsolving mechanism that convenes Internet users in public ways to accomplish tasks collaboratively or independently. A more recent development distinguishes a shift in sourcing intelligence from the crowd from micro to macro tasks, i.e. tasks that are longer, and might require expertise or collaboration [34, 26].

As a consequence of crowdsourcing's online nature, the involved workforce varies in terms of its demographics. Early studies on the topic $[43,21,22]$ revealed that the vast majority of crowdworkers in Amazon Mechanical Turk came from USA and India, and that the workers in USA were predominantly female, while a large portion of crowdworkers from India were male. At the same time, these crowdworkers were in general young and well-educated and tended to have lower incomes and smaller families in comparison to the general population in the US. In line with early findings, more recent studies that have focused on the demographic characteristics of crowdworkers have reported similar results. For example, according to Berg's study in [2], crowdworkers on both Amazon Mechanical Turk and CrowdFlower (now known as FigureEight) were generally well-educated $(53.6 \%$ of the participants involved in their study had college degrees or above) and did part-time crowdtasks (60\% of them held jobs other than crowdwork). In 2018, Difallah, Filatova and Ipeirotis [11] reported that young and well-educated American and Indian crowdworkers continued to be the major workforce on Amazon Mechanical Turk, while the gender distribution among them was more balanced (51\% female and $49 \%$ male). Interestingly, Posch et al. [42] research on crowdworkers' demographics from ten countries indicated that there are significant differences in the reliance on micro-task income and use of micro-task income between the different countries, and that over $40 \%$ of crowdworkers in 7 countries, namely the USA, Brazil, Mexico, India, Indonesia, Philippines and Venezuela, are more likely to use the income from crowdsourcing to cover their basic living expenses.

Moving forwards, the work experiences of crowdworkers have been investigated from a number of vantage points. Lascau et al. [30] suggest that the work environment can affect crowd- 
workers' work efficiency. This proposition is borne out of their recent study which revealed that crowdworkers operating in private spaces displayed good levels of focus while those who worked in a shared space preferred to have a separate area in their home where they could work uninterrupted. Furthermore, Teodoro et al. [50] found that crowdworkers who performed duties outdoors in physical environments (e.g. delivering packages) were more likely to have negative work experiences that could lead to deceptions and physical harm than crowdworkers who worked indoors. Additionally, according to Deng and Joshi [10], crowdworkers at Amazon Mechanical Turk were found to engage more in tasks that were clearly explained, followed evident procedures, entailed meaningful and broad impacts, and allowed higher task-related autonomy. Developing this point further, Jiang et al. [24] posit that perceptions of the rewards gained from tasks are influenced by culture. For their study, the Indian workers regarded self-improvement as the non-monetary rewards, whereas American workers in general valued emotional benefits (e.g. feeling of fulfillment). This does not suggest that monetary rewards do not affect crowdworkers' work experiences. As Ye et al. [64] readily demonstrate, the actual payment gained from tasks can be positively related to the perceived fairness by crowdworkers, which consequently impacts their performance on tasks. For Durward and Blohm [13] another significant factor in the work experience is the motivation to participate in crowdsourcing. In their research, both the intrinsic motivations (e.g. the desire to find interesting tasks) and extrinsic motivations (e.g. the desire to earn money from these tasks) were positively related to the enjoyment perceived by crowdworkers. In their research on the work experiences of Indian crowdworkers, Gupta et al. [17] indicated that workers tended to maintain good reputations in order to participate in well-paid tasks. This is supported by related studies (e.g. [20]) that similarly found crowdworkers with bad reputations earn less than crowdworkers with good reputations. At the same time, this notion of reputation extends beyond the workers. Early crowdsourcing studies [4, 44] have also suggested that crowdworkers would alter their task choices according to the requesters' reputation and were less likely to work for disreputable requesters. For Brawley and Pury [4], job satisfaction of crowdworkers at Amazon Mechanical Turk were affected by dispositional factors (e.g. personality traits), situational factors (e.g. perceived requester support), and interactive factors (e.g. pay satisfaction) while the job satisfaction itself could be used to predict the turnover intentions of crowdworkers. Lastly, Scholarios and Marks [45] discovered that the intrusion of work into private life had a substantial impact on some work-related attitudes even though these workers were relatively individualistic in orientation, highly marketable and unlikely to show attachment to a single organisation. With this in mind, and while acknowledging the similarities between crowdworkers and knowledge workers [19], it is plausible to involve work-life balance in the study of work experience of crowdworkers.

Moving forwards, then, while extant literature provides a thematic schema to examine crowdworkers and their experiences, as detailed above, only a limited number of researchers have focused on crowdworkers in the crowdsourcing context of China. Huo and Zhao [46], for example, revealed that most of the Chinese crowdworkers (59.04\%) in their study are young, between 20 and 25 years old, and that a vast majority of them are well-educated, with bachelor degrees $(58.3 \%)$ or master degrees $(39.48 \%$ ) while only $0.37 \%$ of the workers graduated from junior high school or lower. According to the study of Fei in 2016 [54], approximately three fifths of Chinese crowdworkers (62.95\%) earned more than 2000 CNY (approx. US\$286) per month, which was higher than the general populations in China given the national average of monthly income was around US\$284 in 2016. Alongside this, the study also revealed that Chinese crowdworkers were relatively inexperienced as most of them $(68.64 \%)$ had less than 6 months of crowdwork experience while only a small part of the participants (21.59\%) have been crowdworking over a year.

With regard to the work experience of Chinese crowdworkers, a study from To and Lai has illustrated that Chinese crowdworkers primarily prefer creative tasks and that they would crowdwork with relatively lower payouts than crowdworkers in Western countries [51]. According to Feng and Huang [15], bonus incentives, hobbies and self development were the main motivations of Chinese crowdworkers. Besides, Yang et al. [62] showed that Chinese crowdworkers tend to compete in tasks with fewer participants to increase their opportunities of winning bids, and that they tend to select the tasks with higher expected rewards. In a similar vein, Shi [48] suggested that Chinese crowdworkers in general tend to take easy and well-paying tasks with longer task duration, while workers with higher ratings were more likely to attend challenging tasks for better monetary rewards.

Despite these studies investigating the characteristics of Chinese crowdworkers regarding their motivations, task preferences and operations, limited information has been provided about other aspects of their work experience, such as crowdwork satisfaction and work-life balance. Furthermore, these studies have overlooked the work experiences of a new type of Chinese crowdworkers who have recently been found to be employed by companies to do obligatory crowdwork [55]. These companies, which are dubbed "crowdfarms" by Wang et al. [55], mainly focus on undertaking and doing complex and large crowdsourcing tasks en masse from various crowdsourcing platforms employing salaried employees. Wang et al. [55] based on the discussion with policy makers and crowdsourcing experts have attributed the emergence of crowdfarms to the following three reasons: 1) The changing nature of tasks in the Chinese crowdsourcing platforms - from simple to complex; 2) Favorable government policies such as the the "mass entrepreneurship and mass innovation program" (dazhong chuangye wanzhong chuangxin); and 3) support from Chinese crowdsourcing platforms such as "ZBJ factories" $^{6}$ which provides office spaces for crowdfarms. Further, Wang et al. [55] mention that the emergence of crowdfarms has had significant impact on the remuneration of tasks and work practice of crowdworkers in crowdsourcing context in China.

To address this scholarly gap surrounding the work context of Chinese crowdworkers and the advent of crowdfarm workers,

\footnotetext{
${ }^{6}$ http : //work . zbj . com
} 
our paper compares and contrasts the work experience of solo Chinese crowdworkers to those of crowdfarm workers in light of the changing landscape of crowdsourcing in China.

\section{METHODOLOGY}

To achieve the objectives set forth in this study, we conducted semi-structured telephone interviews with Chinese crowdworkers on one of the largest Chinese crowdsourcing platforms: ZBJ. ZBJ has two main types of tasks. The first is small and relatively low-paying tasks that are called "competition". In competition tasks, requesters need many crowdworkers to work on the same task and everyone can submit their work publicly on the task page for requesters. When their work is accepted, crowdworkers get $80 \%$ of the task reward as compensation, and the remaining $20 \%$ is charged as a service fee by ZBJ. The second type of tasks are relatively large and complex and called "bidding". Although these tasks are open to everyone, requesters who post this type of task usually only need one or more crowdworkers to work for them. In addition, the crowdworkers taking part in the task are required to pay an extra fee besides the $20 \%$ service charge to get the contact information for requesters. This means that the process of bidding is not public as it involves direct communications between crowdworkers and requesters. As the payments of these tasks are often quite substantial, ZBJ usually requires requesters and crowdworkers to sign legal contracts to confirm their respective intention to cooperate. This is done to protect both parties involved in the process. In this study, we wanted to interview a number of key players. To do so, we posted a request for interviews as a "competition" task on ZBJ. This was done after investigating the crowdworker payment for similar type of tasks - usually 50-60 CNY (approx. US\$7 USD to US\$8 USD) on ZBJ platform - an above-average payment of $80 \mathrm{CNY}$ (approx. US\$11 USD) per interview was set.

In total, 48 individuals expressed an interest. After conducting some initial discussions with potential participants we decided to interview all respondents, as most of them (41/48) were experienced crowdworkers who had been crowdworking for more than 6 months, while the rest (7) could share their experiences, from the perspective of a newcomer in crowdsourcing. From the 48 individuals we interviewed, 25 of them crowdworked solo, and 23 worked in crowdfarms. The 25 solo crowdworkers we interviewed were generally young. Most of them (17/25) were between 19 and 35 years old, while the rest of them were under 40 years old. Most solo crowdworkers in our study were generally well-educated. Many of them (16/25) had graduated from vocational schools or other higher education institutions while the rest had high-school degrees. Additionally, a majority of the solo crowdworkers earned more than the general population in China: 21/25 of individuals earned more than $3000 \mathrm{CNY}$ a month (approximately US $\$ 434$ at the time of writing this paper) and only $3 / 25$ earned less than that per month, while the national average monthly income in China was approximately US $\$ 340$ in 2018 [36]. The 23 crowdfarm workers we interviewed were on average 30 years old - 19/23 were either in their late 20 s or early to mid 30 s, while a handful (4/23) were over 40 years of age. The education of the crowdfarm workers was comparable to the education of the solo crowd workers in our study. The clear majority of them (19/23) had graduated from vocational schools or higher education, while only 4 of them had high school or pre-high school. We also found that crowdfarm workers earned significantly more than the general population in China, as well as more than solo crowdworkers. When interviewed, we found that most of them (19/23) earned at least $5000 \mathrm{CNY}$ (approx. US\$714 USD) every month, among which, 10 of them had monthly incomes over $10000 \mathrm{CNY}$ (approx. US\$1449 USD).

In line with extant academic literature in the research area (see Background section), our interviews revolved around the following themes: (1) work environment, (2) tasks, (3) motivation and attitudes, (4) rewards, (5) reputation, (6) crowdwork satisfaction, and (7) work/life balance. For the most part, interviews lasted between 30 and 60 minutes and were conducted in Chinese.

The analysis of our data involved the following stages: (1) familiarization of the data, (2) the development of a thematic framework, and (3) coding of the data. In the familiarization phase, all interview data was transcribed. This was followed by the creation of an initial thematic framework based on (1) prior issues as described in the related literature in Background section and (2) topics that surfaced during the familiarization phase of the categorization of our data. After this phase, the data were independently coded. During the coding stage, we used exact quotes from participants to inductively identify potential themes and patterns within the data, before collating all the relevant coded data extracts within the identified themes.

Once the coding phase was concluded, we continued to refine our initial thematic framework by (1) using the varying agreement rates in individual constructs to identify and resolve issues (e.g. low agreement rates, for instance, indicated that respective codes were defined too broadly and would need clarification), and by (2) collectively reviewing the coded data extracts, and revisiting the whole data-set. This process was undertaken to iron-out any disagreements that arose during the analysis, to ascertain whether the themes "worked" in relation to the data-set, and to identify any additional data within themes. Once we had completed our thematic framework and aligned this with our data-set, we are able to use this in a coherent and accountable manner (e.g.the framework could be used by other researchers and the process replicated).

\section{RESULTS}

\section{Work Environment}

All solo crowdworkers in our study work from home alone because they felt more comfortable, relaxed and were able to work on the tasks whenever they felt like it. This finding is supported by an earlier crowdsourcing study, which discovered that at-home crowdworkers had more flexibility in their crowdwork [23]. However, working from home was not always a positive experience for our participants. Some solo workers complained that they were easily distracted by noises emanating from their surrounding environment. Likewise, the excessive flexibility associated with working at home, often produced difficulties for other participants (6/25). As solo worker P1 explained: 
When I do crowdwork at home, I usually turn on my favorite music to seek for inspiration and get rest whenever I feel tired. You know, it is different from working in company where your boss keeps telling you what to do, when to do and so on. (P1, 26 years old, male)

In contrast to the sentiment of this extract, all of the crowdfarm workers said that they primarily worked in the premises of crowdfarm, but sometimes they worked overtime from home if they had to and if the task at hand didn't require specialized equipment (e.g. a Virtual Reality headset). Crowdfarms are usually based in business premises or in appropriated residential apartments.

When we asked crowdfarm workers how they felt about their physical working environment in the crowdfarm, participants generally had mixed feelings. On one hand, they mentioned that working with colleagues and managers in a crowdfarm helped them do the tasks better than they would on their own because they could ask colleagues for help if needed. On the other hand, they complained that their offices were cramped, sterile, impersonal, with bad air-conditioning. The management of crowdfarms evidently recognizes that this is an issue as some of the crowdfarm workers we interviewed (9/23) mentioned that their company intended to move to new premises soon. This may further improve the productivity of crowdfarm workers as previous studies on various industries (e.g. health care) suggest that a better working environment will help employees offset the exhaustion caused by their workload [9].

\section{Tasks}

All the solo crowdworkers in our study mentioned that they preferred short and easy tasks such as copy-writing and slogan creation, while some of our participants (7/25) said that they would also occasionally attempt more creative tasks (e.g. logo design). This is because, on the one hand, although the payout of short and easy tasks was relatively low, many solo crowdworkers in our study (16/25) were able to complete several of these tasks within a few hours, which meant that they could still earn a considerable amount of money. Those workers who also undertook challenging tasks suggested that it gave them the time to practice and develop their skills. Even if the ensuing work was not accepted, workers saw this as an investment for participating in more challenging and well-paid tasks in the future. This finding is supported by a study with Indian-based Turkers, which demonstrated that crowdworkers could develop their skills considerably through taking part in simple tasks (e.g. drawing bounding boxes) but also developed their skills through taking part in high-level tasks [27].

The major difficulty in carrying out a given task for many solo crowdworkers in our study (17/25) was the requirement and attainment of professional skills and knowledge. An earlier crowdsourcing study demonstrated that the majority of online workforce lacked the expertise to produce quality output [12]. To deal with these difficulties, workers would generally seek help from others and self-study online. P2, a student who was studying design in college, discusses the reasons why he would take challenging tasks and how he dealt with the difficulties in the following extract:
I am still studying how to design so only $10 \%$ to $30 \%$ of my designs were paid. However, I think the important thing to me is that I can practise the skills for my future career in this industry. [...] When I encounter some technical problems or need some new skills, I would usually seek online help, discuss with my classmates who also crowdwork, and sometimes ask my teacher directly. (P2, 19 years old, male)

Even though we found that the solo crowdworkers worked alone from home, this did not necessarily mean there was no collaboration among them. In our study, over $50 \%$ of participants mentioned that they were members of online communities (e.g Wechat groups) that focused around sharing task information and communicating skills. This finding is in line with Gray et al. [16] who similarly claimed that the crowd is a collaborative network. Nonetheless, this was not the case for all participants as some of the solo crowdworkers (4/25) we interviewed intentionally avoided joining such communities as they thought that helping others and sharing task information would lead to excessive competition and low acceptance rate of the tasks. This is largely supported by a crowdsourcing study focusing on the behavior of Chinese crowdworkers, which demonstrated that some Chinese crowdworkers would intentionally choose less popular tasks in order to increase the possibility of their work being accepted [62].

In contrast with solo crowdworkers who procure tasks by themselves, in crowdfarms the workers mentioned that they usually do not have an option to choose which tasks they will work on, and they do the tasks that are assigned to them by their managers. When we asked them about how the crowdfarm's managers procure tasks, they mentioned that usually managers would first act like typical crowdworkers by selecting and bidding for tasks on platforms. However, this procurement was done on behalf of the crowdfarm. After procuring the tasks, managers would request further clarification of the task requirements and set up milestones for the tasks in question. If a task was not decomposable (e.g. creative design tasks), managers would then allocate this task directly to certain crowdfarm workers who specialized in the field. If a task was decomposable (e.g. website building), managers would then turn the task into smaller work units and assign them to different internal teams based on their expertise. During the work process, the manager would also supervise the workers' progress to ensure that everything was running to schedule. After each crowdfarm worker finished the tasks that had been assigned to them, they would then work collaboratively with each other to integrate all parts into a final deliverable and then submit it to the requesters for feedback. P3, the crowdfarm worker who is also a manager shed light on how crowdfarms operate on a day-to-day basis:

The first thing I do is to look for new tasks and bid for proper tasks that I think our company is able to finish.[...] Once I confirm the requirements with requesters, I will decompose the task into different sub-tasks and then allocate the smaller tasks to different team. For example, a designer will be responsible for the UI design and a database engineer for the database [...] We usually have 
a small meeting in the afternoon to make sure everyone is doing his/her work. [...] When every team finish their tasks, we integrate and test the website collaboratively and send it to requesters. " (P3, 40 years old, male)

In contrast to solo workers who preferred short and easy tasks, many crowdfarm workers $(19 / 23)$ hoped that after their manager had considered the work schedule and task complexity, he or she would procure large tasks for them to undertake. When we asked for clarifying information about why this was the case, participants generally thought that compared to small tasks they could earn more money from larger tasks as they could charge more for tasks that were complex and specialized. Indeed, in related research, Yang et al. [62] found that requesters tended to offer more money on tasks needing a higher skill requirement. Moreover, workers explained that the income from large and complex tasks was also more likely to be guaranteed. This is because in $80 \%-90 \%$ of these types of tasks requesters would pay a transaction deposit to ZBJ platform and sign a legal contract with either the crowdfarm or with the manager that represents the crowdfarm in the platform.

With regard to some of the difficulties that crowdfarm workers face, many participants (18/23) said that it was difficult to undertake tasks for requesters who had ill-defined requirements. To solve this problem, crowdfarm workers in our study would commonly help requesters clarify the requirements at the beginning of a project and patiently negotiate with requesters during the project process.

Crowdfarm workers also participated in online communities (e.g. Wechat group) to discuss tasks, platforms and requesters. As well as working with colleagues, many crowdworkers $(10 / 23)$ mentioned that they would also collaborate with other companies and crowdworkers, especially when they were assigned tasks that required expertise from different professions. To complete the tasks with excessive requirements, crowdfarm workers would commonly hand over the part of task that they could not do to a familiar business partner who had expertise in that area and then share the profits with them. Interestingly, few crowdfarm workers (4/23) would also re-crowdsource the undoable parts of their tasks on crowdsourcing platforms for relatively low prices to maximize their own profits. P4 explained how their companies collaborated with others on complex tasks:

In some design tasks, requesters would usually need to print the products. Our company cannot do any printing work but we have been collaborating with a factory that focuses on printing for years. [...] We share the benefits together. (P4, 36 years old, male)

\section{Motivations and attitudes}

When we asked about the motivations for being a crowdworker, the overwhelming majority of solo crowdworkers in our study $(21 / 25)$ suggested that their primary motivation was monetary. However, a large number of them (19/25) also stated that gaining knowledge and acquiring skills, as well as fulfilling personal interests through crowdsourcing, was equally important for being a crowdworker. Furthermore, the solo crowdworkers we interviewed tended to have positive attitudes towards crowdsourcing and mentioned that they would keep crowdworking in the near future (17/25). However, some participants $(6 / 25)$ posited the lack of "doable" tasks for solo crowdworkers was a potential reason for them to stop crowdsourcing. In the main, this is because many tasks on various Chinese crowdsourcing platforms tend to be complex and creative (e.g. product design) or technical (e.g. app development) tasks, which usually require specialized, skilled solo crowdworkers to work together to tackle them [55].

Similarly, the primary motivation of crowdfarm workers was also monetary. For some participants (10/23), their secondary motivation was to gain experience about how these companies worked so that they could start their own businesses in the future. In contrast with solo crowdworkers, only three crowdfarm workers mentioned that gaining knowledge and expertise, or fulfilling personal interests, was their motivation for crowdworking. For the most part, this is because crowdfarm workers were more likely to be assigned obligatory tasks that were consistent with their expertise and the specialization of the crowdfarms and were therefore commonly doing tasks that they have the expertise for.

Compared to solo workers who generally had positive attitudes towards crowdsourcing, many crowdfarm workers $(13 / 23)$ tended to have more neutral attitudes and were not sure whether they would keep working in crowdfarms in the future. This was because many participants thought the payment for crowd tasks was usually unfair for two reasons. First, some requesters would only be willing to pay little for what they required. Second, some crowdfarm workers, especially those who were also managers, mentioned that other crowdfarms were undercutting all bids in order to obtain tasks, which decreased the payout to crowdfarm workers on each task. The following quote from P5, a 30-year-old crowdfarm worker, expresses the concerns and frustrations of crowdfarm workers about the crowdsourcing tasks they have to tackle:

I took a task that looked like they needed a simple online platform with 100k CNY budget (approx. US\$14490 USD). However, it turned out that the requester had no idea about what they wanted. [...] At the end, we received only $1 / 10$ of the money as they thought we did not meet their ever-changing and unrealistic requirements though we had already provided the general framework of the website. [...] I know a company that is also doing crowdwork on ZBJ in our city. Their strategy is to bid with extremely low price to win the tasks. To be honest, if was not because of the platform giving them more opportunities to attract customers with low prices, I seriously doubt if these workers could support themselves with such low profits in tasks. (P5, 30 years old, male)

\section{Rewards}

The solo crowdworkers in our study mentioned that they were satisfied with the immediate monetary reward from crowdsourcing, as they would get paid as soon as requesters accepted the task they completed. When we asked these participants about the importance of the earnings from crowdsourcing, only four suggested that the income from crowdsourcing made little 
contribution to their life. The clear majority of participants mentioned that these earnings improved their life significantly as they used this income to either support their basic living expenses $(5 / 25)$ or to support their hobbies $(16 / 25)$. This is largely in line with findings from an earlier crowdsourcing study, which suggested that $73 \%$ of workers regarded working on Amazon Mechanical Turk as a way to earn extra money, while $19 \%$ of them used this income to 'make ends meet' [43].

Aside from monetary reward, we found that almost all of the solo crowdworkers (20/25) interviewed considered the knowledge and expertise that they gained from the tasks as being a non-monetary reward. P6 explains:

I don't mind doing the video processing task at a payment lower than the market price, as long as it is not too low to support myself, because I think the skills and expertise learned from the task can help me develop my career better. (P6, 27 years old, male)

In contrast with solo crowdworkers, we found that the crowdfarm workers in our study were paid monthly with fixed salaries plus bonuses depending on their performance and their position/rank. In accordance with employees in any other registered Chinese Internet company [58], some participants also indicated that health insurance and pension were also part of their contract in the crowdfarm. However, in contrast with solo crowdworkers who generally appreciated the monetary rewards from crowdsourcing, only a relatively small number of crowdfarm workers (9/23) suggested that the income from crowdsourcing was of great significance to them. When we asked why this was the case, the majority of our participants (14/23) stated that the earning from companies' offline businesses (ie. non-crowdsourcing tasks) was still their major source of income at present whilst 9 of them believed that the earnings from crowdsourcing would soon become their primary source of income as the number of crowd tasks assigned to them by managers were sharply increasing. Instead, what was considered an extremely important gain for them was the non-monetary reward - to establish 'guanxi' (i.e. a Chinese term meaning the interpersonal relationship in which obligation, commitment and exchange of favors are involved [61]) with requesters. This is because, in Chinese business environment, "guanxi" is regarded as an essential for the future transaction and cooperation between counterparties [6]. The trustworthy relationships (guanxi) established through crowd tasks would help crowdfarm workers and managers accumulate customer resources and do further businesses with these regular customers, which would bring more benefits in the long run. P7, a 31 year old crowdfarm worker illustrates why guanxi is important for crowdfarm workers:

If you have a good relationship with requesters, they will pay you quickly once you finish their tasks. Furthermore, they will come back to you for further businesses and these tasks will be much more profitable than the previous ones. [...] (P7, 31 years old, male)

\section{Reputation}

The majority of the solo crowdworkers (19/25) in our study thought that the reputation (e.g. favorable ratings, completion rates) was important for them to increase the possibility of being selected by requesters and therefore earn more money. This is in line with findings from a previous crowdsourcing study which congruently demonstrates that the good reputation of Indian crowdworkers helped them secure well-paid HIT tasks [17].

In order to increase their reputation, all solo crowdworkers mentioned that they would work diligently on tasks and some solo crowdworkers $(9 / 25)$ would also customize their work for requesters based on the characteristics of requesters (e.g. professions, regions). As an indicative example, $\mathrm{P} 8$, a designer who specialized in designing wine bottles, explains how he customises his approach depending on the requester:

I usually check where are the requesters or their companies from when I design wine bottles for them. For example, people in the north of China are more traditional so I would involve more classic elements with red color in my design. However, people from the south China, for example, Shanghai, are less traditional, so I use more modern design concepts with blue or other brighter colors. (P8, 33 years old, male)

The reputation of requesters was similarly understood as being important to many solo crowdworkers in our study (22/25). This is because, compared to disreputable requesters who might pay nothing at the end of the completion of a task, these solo crowdworkers (18/25) would rather work for requesters with better reputation, even if this meant accepting a lower payment. As several participants succinctly put it, "better aught than naught".

When we asked how important reputation is to crowdfarm workers, more than half of crowdfarm workers (12/23) admitted that reputation was important to them, therefore echoing the feelings of solo crowdworkers. That being said, of course, the rest of crowdfarm workers, almost half of them (11/23), did not think reputation mattered so much. Some of these participants explained reputation was not important because their main focus was finishing the tasks assigned to them by managers for payment, rather than to establish their personal reputation and bid for more tasks.

For crowdfarms who intended to maintain their reputation, as well as working diligently like solo workers, crowdfarm workers would also showcase projects they had worked on for requesters on their ZBJ homepage. Interestingly, few crowdfarm workers (4/23) admitted that they would negotiate with requesters and had even given back part of their financial compensation to maintain their reputations when a requester was not satisfied with the work they had done. P9, a manager and worker in a design company, explains how he proactively asked for a favorable rating from a tough requester:

There was a requester that insisted to give us negative feedback [...] At the end, though we had already signed the contract, I had to give him a discount on the price in order to convince him to give us positive feedback. [...]. $(\mathrm{P} 9,31$ years old, male) 
The reputation of requesters also mattered among crowdfarm workers as most of them (14/23) would rather work for a requester with good reputation even though they paid less. However, in contrast to solo crowdworkers who were usually concerned that disreputable requesters would pay nothing, crowdfarm workers thought that these type of requesters would usually be more rigorous and make ever-changing requirements, which often led to unnecessary disputes and costs.

\section{Platform Satisfaction}

Most of the solo crowdworkers in our study only worked for the ZBJ platform (18/25) and the rest of them mentioned that they had previously worked for other Chinese crowdsourcing platforms (e.g. Epwk), but were only working on ZBJ at the moment. Many solo crowdworkers in our study (15/25) suggested that they were satisfied with the ZBJ platform. The crowdwork satisfaction of solo crowdworkers was mainly attributed to the perceived advantages of $\mathrm{ZBJ}$, such as acquiring more tasks, taking advantage of more professional customer services, and enjoying a relatively thorough regulated system. However, these participants were generally dissatisfied with the $20 \%$ service fees charged by ZBJ for each task they completed. This is supported by a previous crowdsourcing study, which illustrated that the 5\%-20\% service fees charged by crowdsourcing platforms would hinder the development of crowdsourcing and reduce the income of crowdworkers [31, 2].

In a similar vein to solo crowdworkers, all crowdfarm workers in our study worked for ZBJ, though many of the crowdfarm workers $(16 / 23)$ had worked for more than 2 platforms before. However, in comparison with solo crowdworkers, we found over half of them (15/23) were unsatisfied with working on ZBJ, among which, 11 of them indicated that they would leave if they found a better crowdsourcing platform in China. In the main, and contrary to solo crowdworker, this was because crowdfarm workers would not only take the burden of service fees but also have to pay extra admission fees to have the opportunity to bid for the tasks they preferred. Moreover, some crowdfarm workers also suggested that ZBJ provided insufficient support to help requesters ascertain task requirements, which significantly increased their time spent in communication with requesters. P10, a programmer from an IT company, details his work experiences:

In some tasks, we are required to pay for the admission fees at the beginning as a ticket to bid with other companies for the tasks and then ZBJ will charge a $20 \%$ service once we have finished the tasks. [...] The communication cost is also a problem. I think ZBJ should at least call the requesters and help them figure out what they want to do before allowing them post the tasks on platform. Otherwise, we have to spend much communicating with requesters just to see if their tasks are suitable for us. It is like a strenuous blind date. (P10, 50 years old, male)

\section{Work-life balance}

We found the solo crowdworkers in our study were generally happy with their work-life balance, as most of them (20/25) suggested that they neither worked overtime on tasks, nor undertook tasks in poor conditions. In addition, most of them $(22 / 25)$ said they had enough spare time and money to take part in leisure activities outside of work. This is mainly because solo crowdworkers, who commonly undertook part-time tasks rather than obligatory tasks, were more likely to arrange the amount of their work and schedule flexibly and, therefore, did not experience the same level of stress felt by those in typical full-time occupation.

When asked about how their families and friends perceived their crowdwork, over half of the solo crowdworkers said they would introduce their crowdwork in a positive way, for example "something for fun", so that their families and friends would usually support them.

Our study also found that in comparison to solo crowdworkers, crowdfarm workers who took on crowdwork as their formal full-time work reported that overall their work-life balance was worse. Many crowdfarm workers (22/23) frequently sacrificed their personal time to work overtime to finish obligatory tasks on time, and many of them $(16 / 23)$ admitted that they had to work when in ill health. P11, a crowdworker from north of China, illustrates how his balance between work and life has suffered:

I often work overtime as the deadline of the task is usually tight. [...] Of course it (work overtime) would affect my personal time. [...] I usually work in ill conditions. Listen to my voice now, I am having a cold and take pills, but I am still working. (P11, 27 years old, male)

As for crowdfarm workers, many of them (15/23) tended to talk less about their crowdwork with their families and friends and simply referred it to as "some online work". For these participants, they did not want to expose families and friends to the various pressures they felt.

\section{DISCUSSION AND CONCLUSION}

Our study compares the work experiences of the Chinese solo crowdworkers and crowdfarm workers. In doing so, it importantly addresses a marked gap in extant CSCW and HCI literature.

Our result suggested that crowdfarm workers work together at relatively formal workplaces, namely business premises or appropriated residential apartments, while solo crowdworkers simply work at home alone. This is because that, in crowdsourcing context in China, crowdfarms are able to obtain more supports from Chinese government and crowdsourcing platforms. For instances, the Chinese governmental program, "mass entrepreneurship and mass innovation program", provides space and monetary easing for small internet companies (See: http://en.drc.gov.cn/2016-04/07/content_ 24350321 . htm) and, more aimingly, ZBJ built 26 business communities in 26 major cities in China for crowdfarms to settle in with cheaper rent, customizable offices and ancillary facilities such as saloon and lounge (See: https://work. zbj .com/). Although these supports mainly focus on attracting crowdfarm, it is our belief that the supports for the work environment of typical solo crowdworkers is also important for the development of crowdsourcing platforms as a previous study on the topic revealed that poor work condition increases the work-related 
exhaustions and disengagement of solo Chinese crowdworkers, which consequently decrease their loyalty and acknowledgement towards the crowdsourcing platform [56]. Additionally, in comparison to solo crowdworkers who enjoyed the at-home flexibility yet got distracted by surrounding environment, we found that working closely in a shared workplace and being supervised by managers help crowdfarm workers do task better than they would on their own. This finding interestingly illustrates that even though crowdsourcing is regarded as a new disruptive business model that deviates from the restrictions of traditional business operations [53], traditional management approaches evidently play a significant role in this new crowdsourcing paradigm as it scales and specializes; a paradigm which requires closer and more frequent internal collaboration and coordination between crowdfarm workers.

Our findings also illustrate that all solo crowdworkers prefer to choose short and easy tasks so as to earn money by finishing many of them, while some of them would also occasionally participate in tasks they found challenging to practice their skills or gain new expertise. This mirrors the findings from previous crowdsourcing studies, which revealed that (1) crowdworkers are able to maximize their earnings through doing a great deal of microtasks [10] and (2) that Chinese solo crowdworkers tend to develop themselves through participating different type of tasks [56]. In contrast, however, crowdfarm workers prefer more complex and larger tasks, although they do tasks that are selected and assigned to them by managers. This finding is understandable as the more complex and larger crowdtasks usually offer better payment [62] and the payment of this type tasks, according to the bidding mechanism of ZBJ (see methodology section), are more likely to be guaranteed by the legal contracts signed with requesters.

Next, we found that the increased complexity of task is the main challenge of solo crowdworkers. This is not surprising given that previous studies in Chinese crowdsourcing context revealed that more and more Chinese individuals and companies start to find crowdsourcing a powerful tool and rely on it to deal with highly complex problems [51]. In contrast, however, the main challenge of crowdfarm workers is not the difficulty of tasks but rather the ill-defined requirements from requesters. This finding illustrate that the unclear task instructions would not only prevent solo crowdworkers from completing tasks $[49,57]$, but also an obstacle for a team of crowdworkers and crowdsourcing organizations. To deal with the challenges in tasks, both crowdfarm workers and solo crowdworkers seek help from others and collaborate with other crowdworkers that they know via social media (e.g. Wechat). Interestingly, in contrast with solo workers, some crowdfarm workers would also hand over parts of tasks that they could not do to their business partners and sometimes re-crowdsource these parts directly to crowdsourcing platforms. Although collaboration between companies is a common practice for small and medium companies in China [32] and, subcontracting tasks means the prices of tasks would be partially controlled by crowdfarms, which, in agreement with Morris et al [35], would lead to the problem in fair payment of crowdtasks.
In addition, our results illustrate that the motivations for solo crowdworkers to crowdwork include monetary rewards (i.e. payment) and non-monetary rewards (i.e. the acquisition of new expertise). This resonates with the findings of previous crowdsourcing studies in China and other countries, which indicate that both extrinsic (e.g. payout) and intrinsic (e.g. learning knowledge) are important motivations for crowdworkers (e.g. $[42,25,2])$. Consequently, the solo crowdworkers would generally have positive attitudes towards remaining in crowdwork although they showed concern about the insufficient number of "doable" tasks, which is due to the changing nature of tasks - from simple to complex [55].

For crowdfarm workers, it is interesting to find that their primary motivation is also monetary yet the most important reward they obtained is non-monetary - the interpersonal relationships with the requesters, or in a Chinese term, Guanxi. This is because, in comparison to western business environment where a relationship follows a successful transaction, in China, the business transactions are often a result of a successful guanxi [39]. A good "Guanxi" binds millions of Chinese firms into a social and business web and it is widely recognized as a key determinant of business performance and market growth [33, 29,5]. Therefore, in our case, the non-monetary reward of crowdfarm workers - Guanxi with requesters - is indeed related to financial benefits as the customer resources accumulated by interpersonal relationships could potentially lead to more business opportunities in the future, which can, of course, lead to more earnings. For the most part, this aligns with the assertions made by O'Neil and Martin [37] who note that crowdsourcing is a relationship-based business and the trust in relationships between crowdworkers and requesters is as important as payments made for tasks. With regard to attitudes, crowdfarm workers are in general more negative than solo crowdworkers. This is because (1) they believe they are paid poorly due to the low rewards of requesters and (2) the price war that exists between competing crowdfarms, which decrease the prices across the Chinese crowdsourcing context in "a race towards the bottom" [55].

Our results suggest that most solo crowdworkers care about and safeguard their reputation in crowdwork as they believe that good reputation will increase the chances of their work being paid by requesters. This is in accordance with previous studies which have similarly shown crowdworkers generally attempt to maintain their reputations $[11,17]$. However, in contrast with these studies and our findings pertaining to solo crowdworkers, our study found that crowdfarm workers have mixed opinions about reputations, as nearly half of them think that a better reputation has little influence on the crowdwork they do. We argue that this is due to the crowdfarm workers being disengaged from the crowdsourcing process (e.g. bidding, procuring the tasks, contacting the requesters etc.) as the foremost responsibility for many of them is to complete the tasks given to them by their managers. With regard to the reputation of requesters, both the solo crowdworkers and crowdfarm wokers in our study mentioned that they prefer to work for requesters with good reputations as they believe requesters with bad reputations are less likely to pay for their works properly and more likely to propose excessive requirements. This 
finding is unsurprising as several crowdsourcing studies have similarly revealed that crowdworkers choose tasks according to the reputation of requesters and are less likely to work for requesters with bad reputations [4, 44].

Further, our findings illustrate that solo crowdworkers are, for the most part, happy with crowdsourcing. In contrast, and in the context of our research, crowdfarm workers tend to have worse crowdwork experiences as a result of service fees. While both solo crowdworkers and crowdfarm workers in our study dislike the $20 \%$ service fees charged by ZBJ, the crowdfarm workers claim that this significantly reduces their earnings. In our discussions with these workers, they propose a bracket-like structure that depends on the complexity of the project and the reputation of the crowdworker and the requestors would be preferable to a flat fee structure that does not account for these factors. In addition, the dissatisfaction of crowdfarm workers relates to an increased communication cost, as almost all participants stated that they had to spend lots of time clarifying and negotiating the detailed requirements with requesters through the inconvenient communication system of ZBJ. Therefore, it is unsurprising these workers also recommend that the ZBJ platform should improve their communication system by providing links to popular social networking software in China, such as Wechat. This finding is supported by previous work from Kingsley et al. that illustrates instant communication with requester and crowdworkers can increase the market power of crowdworkers and has the potential to increase the payouts [28].

Lastly, our findings desmontrate that crowdfarm workers tend to experience higher levels of stress and exhaustion and have less time for leisure and family activities than solo workers. In our opinion, this is, on the one hand, a byproduct of the full-time nature of crowdfarm workers who are assigned obligatory tasks by managers, which means the amount of work and schedule of tasks is not in their hands. On the other hand, this is also attributed to a newly observed 996 work culture among many Chinese IT companies - that is 9am to 9pm, 6 days a week [59]. As the crowdfarms in our study are mainly small Internet companies in nature, their workers are likely to be required to work over 60 hours, which consequently lead to the imbalance of their work and life. This is in contrast to solo crowdworkers who mainly crowdwork part-time and therefore experience much reduced levels of pressure, which illustrates that the different crowdwork nature is the key reason why solo crowdworkers and crowdfarm workers have different work-life balance. In order to prevent the pressures in crowdwork further disturbing their lives, some crowdworkers tend to talk less about their work in front of their families and friends. This finding are supported by previous studies that demonstrate (1) full-time Chinese crowdworkers experienced more work-related fatigues than part-time workers [56] and (2) that workers in different industries commonly cope with the imbalance between work and life by setting up a clear boundary between work time and family time [18].

In sum, then, our study offers one of the first scholarly works to reveal the experiences of crowdfarm workers and how this burgeoning workforce perceive crowdwork differently from solo Chinese crowdworkers. These findings are contextualised through the following thematic schema: (1) work environment, (2) tasks, (3) motivation and attitudes to crowdsourcing, (4) rewards, (5) reputation, (6) crowdwork satisfaction, and (7) work/life balance. This is primarily attributed to the differences in the way these workers engage in crowdsourcing. Based on the novelty of our findings, the present study illustrates that existing approaches to studying the work experiences of typical solo crowdworkers may need to be rethought in order to study these emerging work experiences that are both enabled and impacted by changes in crowdsourcing context such as crowdfarms. Moreover, our study also illustrates that if concomitant platforms want to provide further support to the practice of crowdfarms. For example, since we found that crowdfarm workers mainly work with complex tasks and are in general dissatisfied with the communication tools that the platforms provide, we will suggest that platforms link their communication system to tools that (1) requesters and workers are familiar alike, and (2) support moderated group communication. This can involve extending their current communication systems and integrating them with popular Chinese social meadias such as Wechat through Wechat's API. This is not the case right now as the majority (if not all) of platforms are designed only with the needs of solo crowdworkers in mind and it is, for the most part, quite cumbersome for crowdfarm workers to communicate with requesters especially for complex tasks where constant feedback is needed.

A noteable limitation in the present study is the representativeness of the sample as our participants primarily work for ZBJ platform. Accordingly, crowdworkers on other platforms (e.g. EPWK) might have different experience. In addition, due to the operation in crowdfarms where managers are usually responsible for procuring crowdtasks on behalf of their companies, there are several crowdfarm managers in sample. Although they would also be involved in carrying out the detailed crowdtasks, crowdfarm managers are more likely to perceive and report their work experiences from the perspective of management rather than that of simple crowdfarm workers. Therefore, future work on the topic should perhaps (1) involve more crowdworkers from other Chinese crowdsourcing platforms to increase the representativeness of the sample, and (2) clearly distinguish crowdfarm workers and crowdfarm managers so that further understanding of crowdfarms can be provided through studying crowdwork and experiences from a managerial perspective.

\section{ACKNOWLEDGEMENTS}

This research was supported through the following EPSRC projects: From Human Data to Personal Experience (EP/M02315X/1).

\section{REFERENCES}

[1] Simon à Campo, Vasssilis-Javed Khan, Konstantinos Papangelis, and Panos Markopoulos. 2019. Community heuristics for user interface evaluation of crowdsourcing platforms. Future Generation Computer Systems 95 (June 2019), 775-789. DOI :

http://dx.doi.org/10.1016/j . future. 2018.02.028 
[2] Janine Berg. 2016. Income security in the on-demand economy : findings and policy lessons from a survey of crowdworkers. Technical Report 994906483402676. International Labour Organization. https: //ideas.repec.org/p/ilo/ilowps/994906483402676.html

[3] Daren C. Brabham. 2009. Crowdsourcing the Public Participation Process for Planning Projects. Planning Theory 8, 3 (Aug. 2009), 242-262. DOI: http://dx.doi.org/10.1177/1473095209104824

[4] Alice M. Brawley and Cynthia L.S. Pury. 2016. Work experiences on MTurk: Job satisfaction, turnover, and information sharing. 54 (2016), 531-546. DOI : http://dx.doi.org/10.1016/j.chb.2015.08.031

[5] Peter J. Buckley, Jeremy Clegg, and Hui Tan. 2006. Cultural awareness in knowledge transfer to China-The role of guanxi and mianzi. Journal of World Business 41, 3 (Sept. 2006), 275-288. DOI : http://dx.doi.org/10.1016/j.jwb.2006.01.008

[6] Zhang Chi and Hong Seock-Jin. Guanxi Culture. In The China Business Model. Elsevier, 19-40. DOI : http://dx.doi.org/10.1016/B978-0-08-100750-1.00002-4

[7] Chao-Min Chiu, Ting-Peng Liang, and Efraim Turban. 2014. What can crowdsourcing do for decision support? Decision Support Systems 65 (2014), 40-49. DOI: http://dx.doi.org/10.1016/j.dss.2014.05.010

[8] David Corsar, Peter Edwards, John Nelson, Chris Baillie, Konstantinos Papangelis, and Nagendra Velaga. 2017. Linking open data and the crowd for real-time passenger information. Journal of Web Semantics 43 (March 2017), 18-24. DOI :

http://dx.doi.org/10.1016/j . websem. 2017.02.002

[9] E. Demerouti, A. B. Bakker, F. Nachreiner, and W. B. Schaufeli. 2001. The job demands-resources model of burnout. The Journal of Applied Psychology 86, 3 (June 2001), 499-512.

[10] Xuefei Deng and K.D. Joshi. 2013. Is crowdsourcing a source of worker empowerment or exploitation? understanding crowd workers' perceptions of crowdsourcing career. International Conference on Information Systems (ICIS 2013): Reshaping Society Through Information Systems Design 3 (01 2013), 2350-2359.

[11] Djellel Eddine Difallah, Gianluca Demartini, and Philippe Cudré-Mauroux. 2012. Mechanical Cheat: Spamming Schemes and Adversarial Techniques on Crowdsourcing Platforms. (2012), 26-30.

[12] Mira Dontcheva, Robert R. Morris, Joel R. Brandt, and Elizabeth M. Gerber. 2014. Combining Crowdsourcing and Learning to Improve Engagement and Performance. In Proceedings of the SIGCHI Conference on Human Factors in Computing Systems (CHI'14). ACM, New York, NY, USA, 3379-3388. DOI :

http://dx.doi.org/10.1145/2556288.2557217 event-place: Toronto, Ontario, Canada.
[13] David Durward and Ivo Blohm. 2018. Understanding Job Satisfaction of Crowd Workers: An Empirical Analysis of Its Determinants and Effects. (2018), 13.

[14] Enrique Estellés-Arolas and Fernando González-Ladrón-de Guevara. 2012. Towards an integrated crowdsourcing definition. Journal of Information Science 38, 2 (April 2012), 189-200. DOI : http://dx.doi.org/10.1177/0165551512437638

[15] Xiaoliang Feng and Minxue Huang. 2013. Research on the Solvers' Participating Motivation of Crowdsourcing. 4 (2013). DOI : http: //dx.doi.org/10.14134/j.cnki.cn33-1336/f.2013.04.009

[16] Mary L Gray, Siddharth Suri, Syed Shoaib Ali, and Deepti Kulkarni. 2016. The Crowd is a Collaborative Network. In Proceedings of the 19th ACM Conference on Computer-Supported Cooperative Work \& Social Computing - CSCW'16. ACM Press, San Francisco, California, USA, 134-147. DOI:

http://dx.doi.org/10.1145/2818048.2819942

[17] Neha Gupta, David Martin, Benjamin V. Hanrahan, and Jacki O'Neill. Turk-Life in India. In Proceedings of the 18th International Conference on Supporting Group Work - GROUP '14 (2014). ACM Press, 1-11. DOI: http://dx.doi.org/10.1145/2660398.2660403

[18] Barbara Haddon. 2009. Work-Life Balance: In Search Of Effective Strategies. (2009), 22.

[19] Jeff Howe. 2006. The Rise of Crowdsourcing. Wired (June 2006). https://www . wired.com/2006/06/crowds/

[20] Ying $\mathrm{Hu}$, Yingjie Wang, Yingshu Li, and Xiangrong Tong. 2018. An Incentive Mechanism in Mobile Crowdsourcing Based on Multi-Attribute Reverse Auctions. 18, 10 (2018). DOI : http://dx.doi.org/10.3390/s18103453

[21] Panagiotis G. Ipeirotis. 2010a. Analyzing the Amazon Mechanical Turk marketplace. 17, 2 (2010), 16. DOI: http://dx.doi.org/10.1145/1869086.1869094

[22] Panagiotis G. Ipeirotis. 2010b. Demographics of Mechanical Turk. SSRN Scholarly Paper ID 1585030. Social Science Research Network, Rochester, NY. https: //papers. ssrn. com/abstract $=1585030$

[23] E. Jeffrey Hill, Joseph G. Grzywacz, Sarah Allen, Victoria L. Blanchard, Christina Matz-Costa, Sandee Shulkin, and Marcie Pitt-Catsouphes. 2008. Defining and conceptualizing workplace flexibility. 11, 2 (2008), 149-163. DOI :

http://dx.doi.org/10.1080/13668800802024678

[24] Ling Jiang, Christian Wagner, and Bonnie Nardi. 2015. Not Just in it for the Money: A Qualitative Investigation of Workers' Perceived Benefits of Micro-task Crowdsourcing. In 2015 48th Hawaii International Conference on System Sciences. IEEE, 773-782. DOI : http://dx.doi.org/10.1109/HICSS.2015.98 
[25] N Kaufmann, Thimo Schulze, and Daniel Veit. 2011. More than fun and money. Worker Motivation in Crowdsourcing-A Study on Mechanical Turk. Proceedings of the Seventeenth Americas Conference on Information Systems (01 2011).

[26] Vassillis-Javed Khan, Konstantinos Papangelis, Ioanna Lykourentzou, and Panos Markopoulos (Eds.). 2019. Macrotask Crowdsourcing: Engaging the Crowds to Address Complex Problems. Springer International Publishing, Cham. DOI:

http://dx.doi.org/10.1007/978-3-030-12334-5

[27] Shashank Khanna, Aishwarya Ratan, James Davis, and William Thies. 2010. Evaluating and Improving the Usability of Mechanical Turk for Low-income Workers in India. In Proceedings of the First ACM Symposium on Computing for Development (ACM DEV'10). ACM, New York, NY, USA, 12:1-12:10. DOI : http://dx.doi.org/10.1145/1926180.1926195 event-place: London, United Kingdom.

[28] Sara Kingsley, Mary-Louise Gray, and Siddharth Suri. 2014. Monopsony and the Crowd: Labor for Lemons? (2014). DOI : http://dx.doi.org/10.2139/ssrn. 3257857

[29] Eike A Langenberg. Guanxi and business strategy: theory and implications for multinational companies in China; with 4 tables. Physica-Verl. OCLC: 845163084.

[30] Laura Lascau, Sandy J. J. Gould, Anna L. Cox, Elizaveta Karmannaya, and Duncan P. Brumby. Monotasking or Multitasking: Designing for Crowdworkers' Preferences. In Proceedings of the 2019 CHI Conference on Human Factors in Computing Systems - CHI '19 (2019). ACM Press, 1-14. DOI : http://dx.doi.org/10.1145/3290605.3300649

[31] Ming Li, Jian Weng, Anjia Yang, Wei Lu, Yue Zhang, Lin Hou, Jia-Nan Liu, Yang Xiang, and Robert H. Deng. 2019. CrowdBC: A Blockchain-Based Decentralized Framework for Crowdsourcing. 30, 6 (2019), 1251-1266. DOI :

http://dx.doi.org/10.1109/TPDS.2018.2881735

[32] Xiangfeng Liu. 2008. SME Development in China: A Policy Perspective on SME Industrial Clustering. 5 (2008), 37-68. http://www . eria. org/SME\%20Development\% 20in\%20China_A\%20Policy\%20Perspective\%20on\%20SME\% 20Industrial\%20Clustering.pdf

[33] Yadong Luo. Guanxi and Business. Asia-Pacific Business Series, Vol. 1. WORLD SCIENTIFIC. DOI : http://dx.doi.org/10.1142/4258

[34] Ioanna Lykourentzou, Vassillis-Javed Khan, Konstantinos Papangelis, and Panos Markopoulos. 2019. Macrotask Crowdsourcing: An Integrated Definition. Springer International Publishing, Cham, 1-13. DOI : http://dx.doi .org/10.1007/978-3-030-12334-5_1

[35] Meredith Ringel Morris, Jeffrey P. Bigham, Robin Brewer, Jonathan Bragg, Anand Kulkarni, Jessie Li, and Saiph Savage. 2017. Subcontracting Microwork. In Proceedings of the 2017 CHI Conference on Human
Factors in Computing Systems - CHI '17. ACM Press, 1867-1876. DOI :

http://dx.doi.org/10.1145/3025453.3025687

[36] National Bureau of Statistics of China. 2018. Annual data 2018. http:

//data. stats.gov. cn/english/easyquery.htm?cn=c01. (2018). Accessed June 4, 2019.

[37] Jacki O’Neill and David Martin. 2013. Relationship-Based Business Process Crowdsourcing? In Human-Computer Interaction - INTERACT 2013, Paula Kotzé, Gary Marsden, Gitte Lindgaard, Janet Wesson, and Marco Winckler (Eds.). Vol. 8120. Springer Berlin Heidelberg, 429-446. DOI :

http://dx.doi.org/10.1007/978-3-642-40498-6_33

[38] Konstantinos Papangelis, Alan Chamberlain, and Hai-Ning Liang. 2016. New directions for preserving intangible cultural heritage through the use of mobile technologies. In Proceedings of the 18th International Conference on Human-Computer Interaction with Mobile Devices and Services Adjunct - MobileHCI '16. ACM Press. DOI :

http://dx.doi.org/10.1145/2957265.2962643

[39] Seung Ho Park and Yadong Luo. 2001. Guanxi and organizational dynamics: organizational networking in Chinese firms. 22, 5 (2001), 455-477. DOI :

http://dx.doi.org/10.1002/smj . 167

[40] Marion K. Poetz and Martin Schreier. 2012. The Value of Crowdsourcing: Can Users Really Compete with Professionals in Generating New Product Ideas? Journal of Product Innovation Management 29, 2 (2012), 245-256. DOI : http://dx.doi.org/10.1111/j.1540-5885.2011.00893.x

[41] Lisa Posch, Arnim Bleier, Fabian Flöck, and Markus Strohmaier. 2018a. Characterizing the Global Crowd Workforce: A Cross-Country Comparison of Crowdworker Demographics. arXiv preprint arXiv:1812.05948 (2018).

[42] Lisa Posch, Arnim Bleier, Fabian Flöck, and Markus Strohmaier. 2018b. A Cross-Country Comparison of Crowdworker Motivations. (2018). http://arxiv.org/abs/1711.03115

[43] Joel Ross, Lilly Irani, M. Six Silberman, Andrew Zaldivar, and Bill Tomlinson. Who are the crowdworkers?: shifting demographics in mechanical turk. In Proceedings of the 28th of the international conference extended abstracts on Human factors in computing systems - CHI EA '10 (2010). ACM Press, 2863. DOI : http://dx.doi .org/10.1145/1753846.1753873

[44] Gordon Schmidt and William Jettinghoff. 2016. Using Amazon Mechanical Turk and other compensated crowdsourcing sites. 59 (2016). DOI : http://dx.doi .org/10.1016/j . bushor.2016.02.004

[45] Dora Scholarios and Abigail Marks. 2004. Work-life balance and the software worker. 14 (2004), 54-74. DOI : http://dx.doi.org/10.1111/j.1748-8583.2004.tb00119.x 
[46] Huo Shengping and Zhao Wei. 2019. The Effect Mechanism of Witkey' s Creative Self - Efficacy on Their Innovation Behavior on the Network Innovation Platform. 35, 2 (2019), 34-46. DOI : http://dx.doi.org/10.1207/s15327906mbr0102_10

[47] Shengping Huo, Zheng Chijian, and Hailang Tu. 2017. On the Research of Intelligent Coupling and Coordination between Employers and Witkey on the Crowd Innovation Network Platform. Journal of Xiangtan University 41, 1 (Jan. 2017), 94-101. D0I : http://dx.doi.org/0.13715/j.cnki.jxupss.2017.01.017

[48] Lei Shi. 2012. Factors Affecting Solvers to Participate in Crowdsourcing: An Empirical Study from China. (2012).

[49] M. Six Silberman, Joel Ross, Lilly Irani, and Bill Tomlinson. Sellers' problems in human computation markets. In Proceedings of the ACM SIGKDD Workshop on Human Computation - HCOMP '10 (2010). ACM Press, 18. DOI :

http://dx.doi.org/10.1145/1837885.1837891

[50] Rannie Teodoro, Pinar Ozturk, Mor Naaman, Winter Mason, and Janne Lindqvist. The motivations and experiences of the on-demand mobile workforce. In Proceedings of the 17th ACM conference on Computer supported cooperative work \& social computing CSCW'14 (2014). ACM Press, 236-247. DOI : http://dx.doi.org/10.1145/2531602.2531680

[51] W. To and L. S. L. Lai. 2015. Crowdsourcing in China: Opportunities and Concerns. 17, 3 (2015), 53-59. DOI : http://dx.doi.org/10.1109/MITP.2015.47

[52] UNSW Australia Business School, Daniel Schlagwein, Niels Bjorn-Andersen, and Copenhagen Business School. 2014. Organizational Learning with Crowdsourcing: The Revelatory Case of LEGO. Journal of the Association for Information Systems 15, 11 (Nov. 2014), 754-778. DOI :

http://dx.doi.org/10.17705/1jais.00380

[53] Florian Waldner and Marion Poetz. 2015. Crowdsourcing Business Model Innovation.

[54] Liwei Wang, Jian Tian, and Yi Xu. 2015. Research on the Influential Factor and Their Mechanism of Online Innovation Contest Performance. (2015). http://kreader . cnki .net/Kreader/CatalogViewPage . aspx? $\mathrm{dbCode}=\mathrm{cdmd} \&$ filename $=1016736048$.

[55] Yihong Wang, Konstantinos Papangelis, Ioanna Lykourentzou, and Vassilis-Javed Khan. 2019. The
Changing Landscape of Crowdsourcing in China: From Individual Crowdworkers to Crowdfarms. The 22nd ACM Conference on Computer-Supported Cooperative Work and Social Computing (CSCW'19) (2019). DOI : http://dx.doi.org/10.1145/3311957.3359469

[56] Yihong Wang, Konstantinos Papangelis, Ioanna Lykourentzou, Hai-Ning Liang, Irwyn Sadien, Evangelia Demerouti, and Vassilis-Javed Khan. 2020. In Their Shoes: A Structured Analysis of Job Demands, Resources, Work Experiences, and Platform Commitment of Crowdworkers in China. 4 (2020), 40. DOI : http://dx.doi.org/10.1145/3375187

[57] Meng-Han Wu and Alexander J Quinn. 2017. Confusing the Crowd: Task Instruction Quality on Amazon Mechanical Turk. (2017), 10.

[58] Bingqing Xia. 2014. Labour in the Chinese internet industries. (2014), 263. https://pdfs. semanticscholar. org/ea5d/ce14122bfd061f652fc7dce523d4bb7b928d.pdf

[59] Li Xiaotian. 2020. The 996 ICU Movement in China: Changing Employment Relations and Labour Agency in the Tech Industry. (2020). http://bit.1y/2Rs5o0m

[60] Xinhua. 2015. China to explore new models to boost innovation, entrepreneurship - Business Chinadaily.com.cn. (Sept. 2015). http://www. chinadaily. com.cn/business/2015-09/26/content_21989332.htm

[61] Fang Yang. 2011. The Importance of Guanxi to Multinational Companies in China. 7, 7 (2011), p163. DOI : http://dx.doi.org/10.5539/ass.v7n7p163

[62] Jiang Yang, Lada A Adamic, and Mark S Ackerman. 2008. Crowdsourcing and Knowledge Sharing: Strategic User Behavior on Taskcn. (2008), 10.

[63] Xun Yang. 2016. China highlights 'crowd platforms' to encourage entrepreneurship and innovation - People's Daily Online. (March 2016). http://en.people.cn/n3/2016/0309/c98649-9027466.html

[64] Teng Ye, Sangseok You, and Lionel P Robert Jr. 2017. When Does More Money Work? Examining the Role of Perceived Fair- ness in Pay on the Performance Quality of Crowdworkers. (2017), 10.

[65] Sharon Zhou, Melissa Valentine, and Michael S. Bernstein. In Search of the Dream Team: Temporally Constrained Multi-Armed Bandits for Identifying Effective Team Structures. In Proceedings of the 2018 CHI Conference on Human Factors in Computing Systems - CHI '18 (2018). ACM Press, 1-13. DOI : http://dx.doi.org/10.1145/3173574.3173682 